\title{
Análisis de dos modelos de actividad basados en los procesos de autorregulación para la aplicación del concepto de enlace de hidrógeno a la interpretación de propiedades físicas de las sustancias
}

Juan Antonio Llorens-Molina ${ }^{a}$, Jesús $\mathbf{M}^{\mathbf{a}}$ Llorens de Jaime $^{\mathrm{b}}$

${ }^{a}$ Grupo IEMA (ICE Universidad Politécnica de Valencia), e-mail: juallom2@qim.upv.es

${ }^{b}$ Facultad de Ciencias Políticas y Sociología (Universidad Complutense de Madrid), e-mail: jmllorens@estumail.ucm.es

\begin{abstract}
The self-regulation processes are a keyfactor in learning autonomy development. These processes involve a learning activities design which enhances the way of giving information and feed-back. The application of hydrogen bond concept in order to interpret physical properties has been evaluated in this work. The developed activity consisted of observing and comparing the viscosity of different alcohols. For this purpose, the effect in learning achievement of two activity models based on different cognitive processes, followed by the same type of feed-back, has been comparatively analyzed. The results have shown how carrying out these activities improves the learning. It is particularly significant when the activity involves a selfregulation process based on the analysis of given information after asking for an explanation of the proposed experiment.
\end{abstract}

Keywords: assessment, self-regulation, feed-back, learning activity, experiment, physical properties, viscosity.

\footnotetext{
Resumen

Los procesos de autorregulación son un factor clave en el desarrollo de la autonomía en el aprendizaje. Dichos procesos implican un diseño de las actividades que ponga un especial énfasis en el modo de proporcionar la información y la retroalimentación. En este trabajo se ha evaluado la aplicación del concepto de enlace de hidrógeno a la interpretación de las propiedades físicas de las sustancias. La actividad desarrollada consistió en observar e interpretar la diferencia de viscosidad entre diferentes alcoholes. Con este objetivo, se ha analizado comparativamente la influencia en el aprendizaje de dos modelos de actividad que se distinguen por los procesos cognitivos implicados, proporcionando después el mismo tipo de retroalimentación. Los resultados obtenidos han mostrado cómo la realización de estas actividades trae consigo una mejora en el aprendizaje. Esta es particularmente significativa cuando la actividad implica un proceso de autorregulación basado en el análisis de la información proporcionada tras el planteamiento inicial del problema.
} 
Palabras clave: evaluación, autorregulación, retroalimentación, actividad de aprendizaje, experimento, propiedades fisicas, viscosidad.

\section{Introducción}

\section{Autorregulación y retroalimentación como elementos básicos de una orientación constructivista del aprendizaje.}

La responsabilidad del estudiante acerca de su propio aprendizaje puede considerarse un elemento básico en cualquier propuesta educativa de orientación constructivista (Driver, 1986). En efecto, si el aprendizaje es concebido como un proceso continuo de reestructuración cognitiva en el que la interacción social juega un papel clave (Vygotsky, 1993) el desarrollo de la autonomía del estudiante se convierte en el principal objetivo de cualquier tarea docente (González, 2006). Este desarrollo viene condicionado por múltiples factores e implica profundos cambios en nuestra realidad educativa a diferentes niveles: desde la actividad cotidiana en aulas y laboratorios a decisiones de índole organizativa. Desde una perspectiva centrada en el diseño y evaluación de las actividades de aprendizaje en la que se sitúa este trabajo, metacognición y autorregulación pueden considerarse procesos fundamentales en una concepción del aprendizaje como construcción de conocimiento, más aún, cuando éste se contempla como tarea automotivada y que se desarrolla de modo permanente a lo largo de la vida (Martín, 2012).

Ello es especialmente relevante si se considera que aprender no es tanto incorporar nuevos conocimientos y habilidades, como modificar, desarrollar y, en ocasiones, transformar radicalmente conocimientos y enfoques metodológicos preexistentes. De este modo, la movilización de dichas concepciones y su contraste con la nueva información, transmitida de forma diversa a través de la actividad docente (materiales de aprendizaje, colaboración con pares, profesorado) o del contexto social (medios), daría lugar a la reestructuración cognitiva a la que anteriormente se aludía.

Son múltiples las estrategias didácticas que pueden favorecer los procesos de autorregulación y, particularmente, en cursos introductorios de química en la educación superior (Coppola, 1995). Algunas de las más importantes serían:

- La propuesta y discusión de ejemplos y contraejemplos de conceptos, sobre todo si son contraintuitivos.

- La realización de actividades que proporcionen a los estudiantes oportunidades para explicitar sus interpretaciones y construir argumentaciones para defenderlas.

- La reflexión crítica sobre la validez de las analogías y metáforas introducidas en la construcción de los conceptos científicos.

- La organización, selección y comprensión de la información procedente de múltiples fuentes.

- La integración mediante el establecimiento de relaciones y la aplicación a nuevos contextos del conocimiento adquirido.

Un elemento clave a la hora de diseñar actividades que favorezcan la autorregulación es el modo y momento en que se presenta la información en la secuencia de actividades. Un

2016, Universitat Politècnica de València 
proceso de autorregulación típico en el aprendizaje científico consistiría, por ejemplo, en el planteamiento de un hecho experimental problemático, proporcionando posteriormente información que lleve al estudiante a contrastar ésta con sus expectativas e ideas iniciales. Una retroalimentación posterior mediante la discusión en el aula o a través de cualquier otro medio completaría la citada secuencia. Esta estrategia es notablemente distinta de otras propuestas de carácter más abierto (pero no siempre más eficaces) dónde la respuesta al planteamiento de problemas consiste en la búsqueda espontánea de información.

En el diseño de este tipo de actividades la retroalimentación es un elemento clave, ya que implica un enfoque de la evaluación como un tipo de actividad integrada en el proceso de aprendizaje. Es importante precisar, no obstante, que la retroalimentación no necesariamente produce autorregulación (García-Jiménez, 2015) aunque sea una condición para lograrla, pues no consiste exclusivamente en proporcionar la respuesta correcta, incluso cuando ésta se justifica detalladamente, sino en incidir en el modo en que el estudiante se enfrenta a la cuestión planteada. Aquí radica precisamente la clave de este trabajo, ya que los dos modelos de actividad comparados se distinguen precisamente en la inclusión o no de información cuya contrastación con el hecho experimental observado pueda generar autorregulación previamente a la retroalimentación proporcionada al final del proceso.

Otro aspecto importante a la hora de valorar la importancia de la retroalimentación en el aprendizaje es la gran oportunidad que brindan las nuevas tecnologías para que sea más eficaz, rápida e interactiva. (Maldonado, 2009). Las herramientas colaborativas permiten no solamente una retroalimentación inmediata, sino más enriquecedora al diversificar las fuentes de las misma, concediendo un especial protagonismo a la interacción entre iguales a través de foros, wikis, etc.

\section{La interpretación de las propiedades físicas a partir de la estructura molecular: un importante objetivo en la introducción a la química orgánica.}

Uno de los pilares básicos (y frecuentemente olvidado) en la didáctica de las ciencias es la fundamentación epistemológica. Más allá de los criterios pedagógicos de carácter general, el elemento esencial de la transposición didáctica en el aprendizaje científico es el origen y evolución del propio conocimiento, de sus dificultades intrínsecas, interpretables habitualmente a la luz del contexto social, histórico y científico en que dicho conocimiento se generó. En este sentido, uno de los obstáculos más relevantes en la comprensión del esquema conceptual relacionado con la estructura de la materia y sus transformaciones es la adecuada coordinación entre los diferentes niveles de descripción: macroscópico, simbólico y atómico-molecular, tal como se muestra en la figura 1, con el ejemplo del agua. 


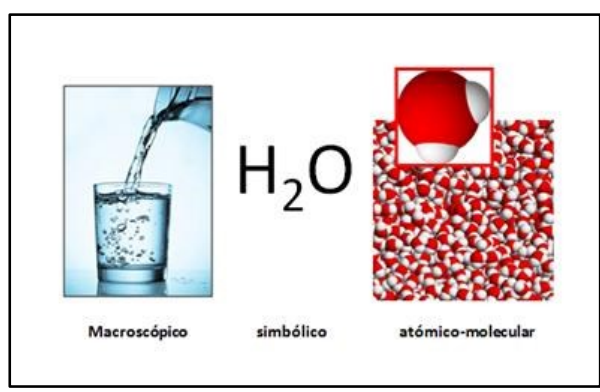

Figura 1. Niveles de descripción de la materia.

Esta dificultad ha sido ampliamente estudiada en la literatura didáctica (Llorens, 1991; Gilbert, 2009) y puede ser abordada desde un punto de vista epistemológico teniendo en cuenta el entramado conceptual que subyace en los conocimientos básicos de la química. En efecto, conceptos tales como sustancia pura, cambio químico o físico, elemento, compuesto, átomo, molécula, etc., son frecuentemente objeto de una escasa comprensión, muchas veces ignorada cuando se pretende construir conocimiento sobre la base de los mismos (Llorens, 1991; Pozo, 1991; Taber, 2001; Kind, 2014; Talanquer, 2009). A modo de ejemplo, la figura 2 muestra algunas interpretaciones gráficas elaboradas por estudiantes de $1^{\text {er }}$ curso de un grado de ingeniería en torno a la evaporación del etanol, en las que se manifiestan claramente las citadas dificultades.
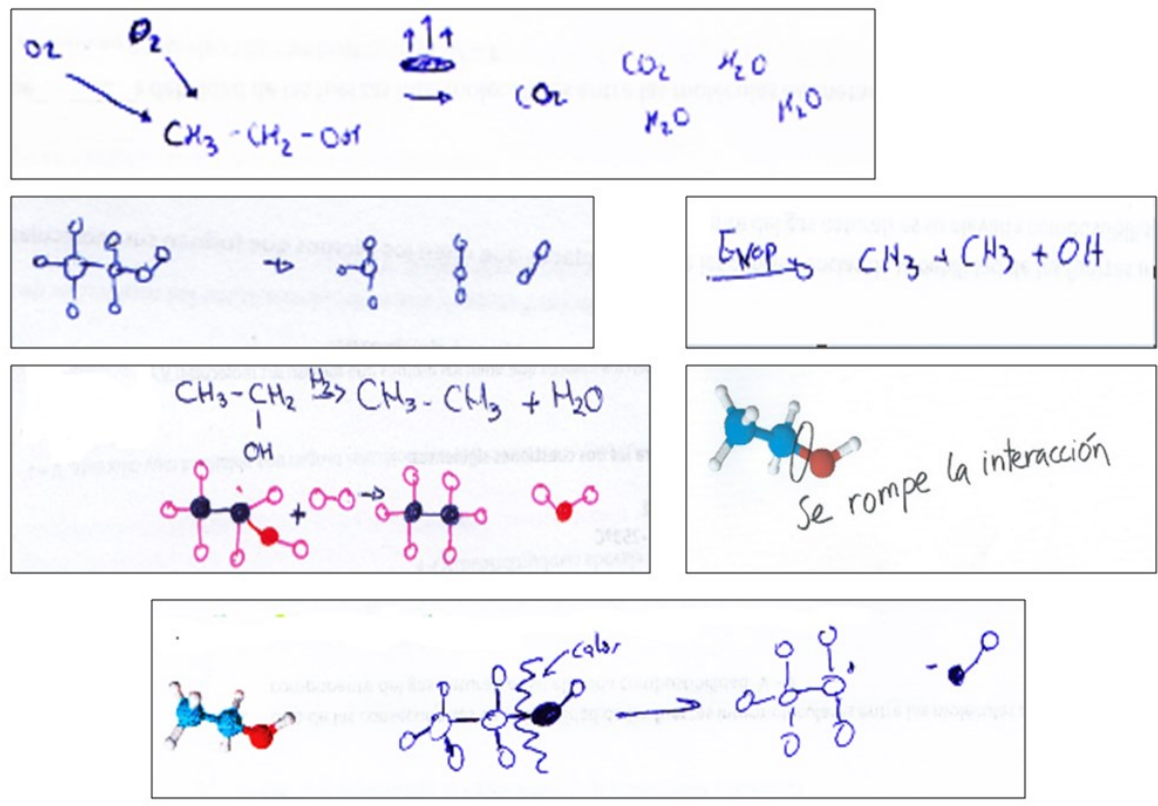

Fig.2 Algunas representaciones a nivel atómico-molecular de la evaporación del etanol elaborados por estudiantes de un primer curso de ingeniería.

Asimismo, la figura 3 muestra también dos ejemplos de respuestas elaboradas por el mismo colectivo de estudiantes a tareas en las que se solicita relacionar las propiedades físicas con las interacciones moleculares.

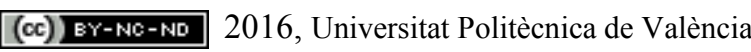




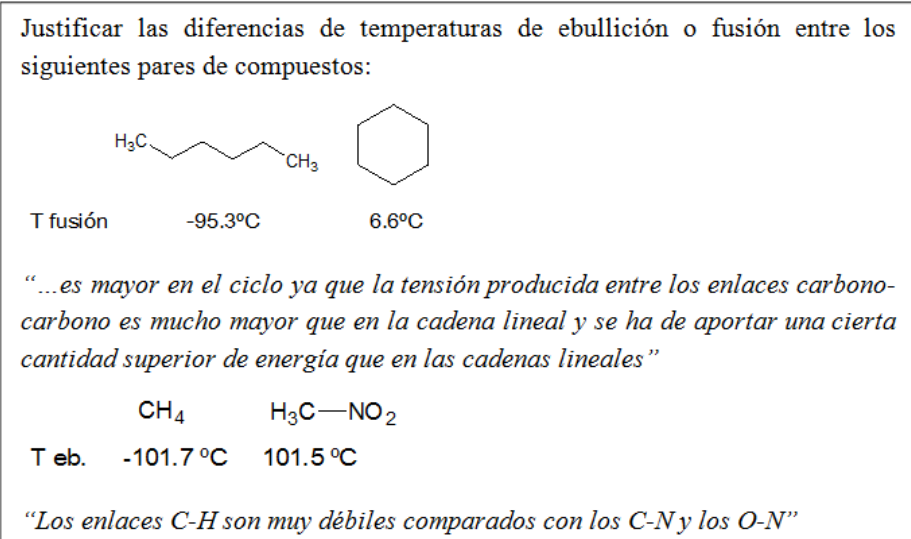

Fig. 3. Ejemplos de interpretaciones alternativas de las diferencias entre las propiedades fisicas de las sustancias

Puede pues afirmarse que errores conceptuales tales como la interpretación de los cambios de fase como ruptura de enlaces covalentes o el uso ambiguo del término "estabilidad" referido tanto a los cambios físicos como químicos, etc., poseen una notable incidencia incluso entre el alumnado universitario. Tal como menciona Talanquer (2009): "Una significativa proporción de estudiantes, desde la escuela secundaria hasta graduados en química, no tiene un claro sentido del origen fisico de las fuerzas entre las partículas en las sustancias químicas, ni reconocen el papel fundamental de estas fuerzas en la determinación de las propiedades fisicas y químicas de los materiales".

Son diversas las causas atribuidas a estos hechos por diferentes autores. Cabe destacar:

- El uso confuso y contradictorio del lenguaje relacionado con las interacciones moleculares (Kind, 2004)

- La inadecuada simplificación de ciertos modelos y representaciones utilizados al describir la estructura atómico-molecular de la materia (Özmen, 2004; Nahum, 2007; Kronik, 2008; Galagovsky, 2009; Nahum, 2010).

- La escasa atención concedida al estudio de las fuerzas intermoleculares en la introducción al enlace químico, así como la excesiva focalización en el concepto de molécula al introducir los conceptos básicos de química, en detrimento de otros tipos de organización microscópica de la materia como las estructuras reticulares y macromoleculares, fundamentales a la hora de interpretar las propiedades de los materiales más comunes (Llorens, 1991; Tarhan, 2008; Schmidt, 2009)

Dentro de las interacciones moleculares, el enlace de hidrógeno posee especial importancia por su influencia en las propiedades físicas en ejemplos tan notorios como el papel del agua en la naturaleza y en los procesos biológicos, en la estructura de las proteínas o en las propiedades mecánicas de materiales como el nilón. Sin embargo, tal como indica Henderleiter (2001), su aplicación a la interpretación de propiedades físicas no está exenta de dificultades. Por este motivo cabe concederle una especial atención en un curso introductorio a la química orgánica en cualquier grado, sobre todo en las bioingenierías.

2016, Universitat Politècnica de València

Congreso In-Red (2016) 
Desde el punto de vista didáctico el concepto de enlace de hidrógeno presentado como ejemplo de interacción particularmente intensa entre dipolos, originada por la elevada electronegatividad de F, O y N está siendo cuestionado (Weinhold, 2014). No obstante, desde la perspectiva de este trabajo ha sido asumido tal como se propone en los textos de bachillerato y en los manuales universitarios más generalizados en nuestro entorno educativo -(Petrucci, 2010), por ejemplo-.

Un experimento particularmente interesante relacionado con los efectos del enlace de hidrógeno en las propiedades de los materiales es introducido en el proyecto Salters Advanced Chemistry (Burton, G.,1994) y muestra la influencia en la viscosidad de la distinta capacidad de formación de enlaces de hidrógeno en el 1-propanol, el 1,2propanodiol y el 1,2,3-propanotriol (glicerina). El interés de este experimento se justifica por: (1) el enlace de hidrógeno se emplea para justificar una propiedad -la viscosidaddiferente a las que se utilizan habitualmente: temperaturas de cambio de estado y solubilidad, (2) por la importancia de esta propiedad en el contexto de los estudios de ingeniería.

\section{Objetivos}

1. Desde el punto de vista metodológico, desarrollar y aplicar un modelo para el diseño y evaluación de actividades que favorezcan la autorregulación en el aprendizaje, potencialmente aplicable a otros contenidos del curso, que consisten esencialmente en:

a. Presentación de un hecho experimental potencialmente problemático.

b. Análisis del mismo a través de dos alternativas:

- Contrastación con información proporcionada acerca del experimento planteado, pero que no da respuesta directa a sus resultados.

- Búsqueda espontánea de información que dé respuestas a dichos resultados.

c. Retroalimentación final.

2. Evaluar en qué medida la realización o no de estas actividades permite mejorar la capacidad para explicar otras propiedades físicas a partir de las interacciones moleculares. Al mismo tiempo, se analizan comparativamente las dos alternativas anteriormente indicadas en el diseño de la actividad.

3. Proponer un modelo de rúbrica que permita evaluar de modo riguroso la actividad, en el contexto de la experimentación educativa. Sus descriptores serían después el fundamento para la definición y, sobre todo, aplicación de los utilizados en rúbricas aplicadas posteriormente en actividades similares, dentro de la docencia ordinaria.

\section{Desarrollo de la innovación}

\section{Contexto y planificación}

El estudio de las interacciones moleculares es uno de los temas introductorios que forman parte de la unidad didáctica 1 , dedicada a la iniciación a la química orgánica, dentro de la 
asignatura Fundamentos Químicos para Ciencia y Tecnología de Alimentos, del $1^{\text {er }}$ curso de dicho grado en la ETSIAMN, de la Universidad Politécnica de Valencia. La innovación fue experimentada en los dos grupos, A y B, con un total de 94 estudiantes matriculados. Cabe precisar que, a efectos de evaluar la influencia de la actividad en el aprendizaje, las puntuaciones de los repetidores fueron excluidas por la posibilidad de conocer su contenido de cursos anteriores.

Dentro de las tareas habituales de evaluación utilizadas en esta unidad se efectúa una prueba semanal de autoevaluación online, con retroalimentación inmediata mediante los comentarios, a través de la herramienta "exámenes" de PoliformaT (plataforma online de soporte a la docencia, basada en SAKAI, de la UPV). En el marco de estas actividades se introdujo en la primera prueba un conjunto de cuestiones encaminadas a evaluar los conocimientos iniciales (prueba inicial) en torno a tres cuestiones potencialmente problemáticas:

a) Distinción entre enlace covalente (intramolecular) con las interacciones moleculares.

b) La vinculación entre los cambios químicos y la ruptura y formación de enlaces covalentes, así como entre los cambios físicos y la modificación de las interacciones moleculares.

c) Concepto de polaridad y las moléculas y su influencia en las interacciones moleculares.

Esta prueba inicial es decisiva a la hora de interpretar los resultados obtenidos, ya que permite una evaluación previa de las posibles diferencias entre los distintos colectivos considerados: estudiantes que realizan cada uno de los dos modelos de actividad y los que no la realizan. A partir de ahí, el proceso seguido queda expuesto en el diagrama de la figura 4. Tras la prueba inicial, se impartió el tema dedicado a las interacciones moleculares con una metodología basada principalmente en la discusión de propiedades de sustancias de interés tecnológico o relacionadas con la vida cotidiana, incluyendo actividades de consolidación y ampliación basadas en grabaciones polimedia (sistema diseñado en la UPV para la creación de contenidos multimedia como apoyo a la docencia) a partir del material proporcionado en "contenidos" de poliformaT. Al final de la unidad se llevó a cabo la secuencia de actividades propuesta, según el modelo que queda también descrito en la figura 4.

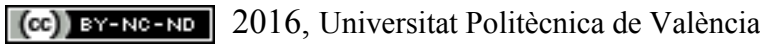




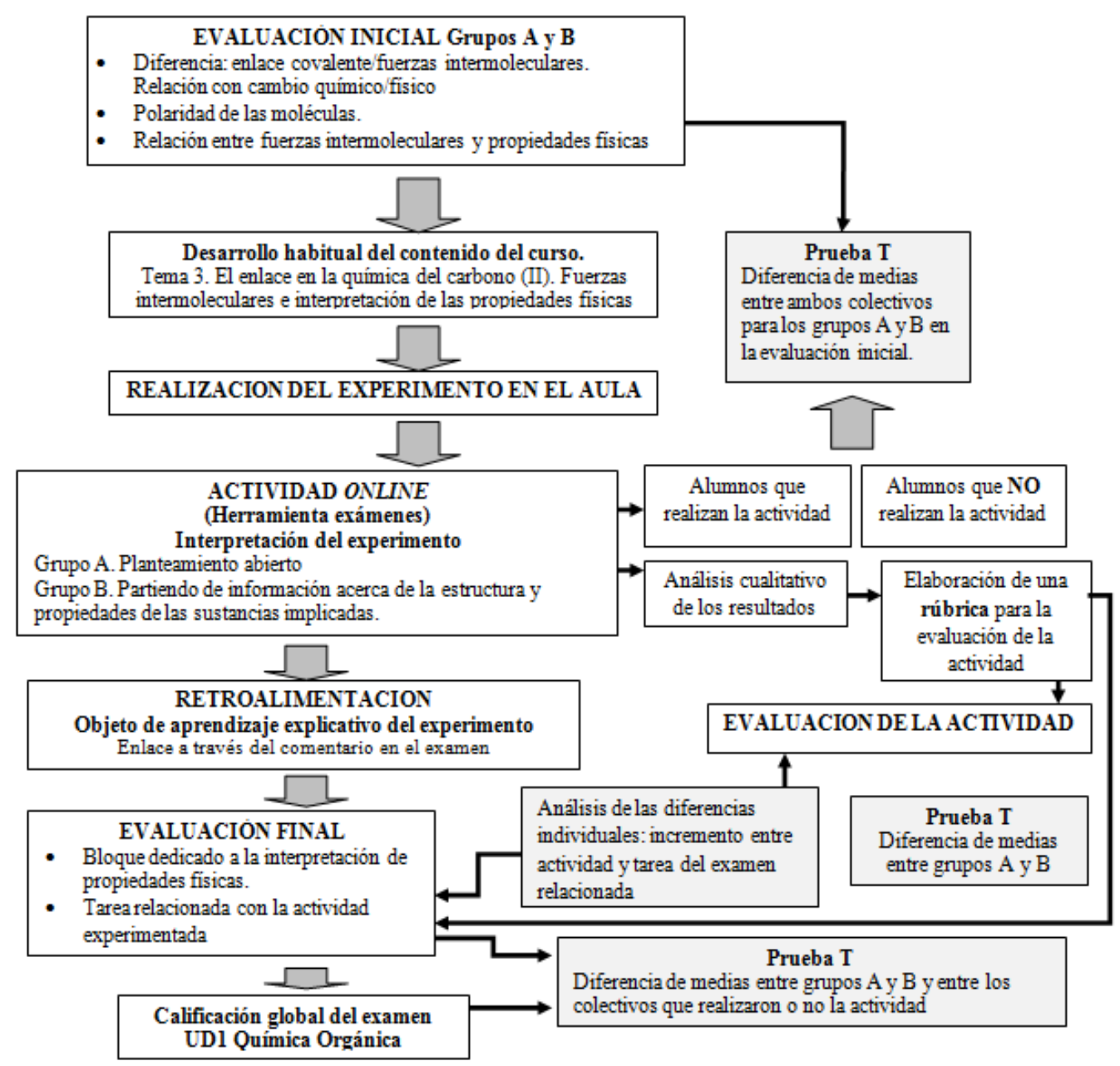

Fig. 4. Diseño de la experimentación

La actividad se inicia en el aula con una demostración experimental que pone en evidencia las diferencias de viscosidad entre el 1-propanol, 1,2-propanodiol y 1,2,3-propanotriol. Tras esta demostración no se llevó a cabo ningún tipo de aclaración o discusión; simplemente, los estudiantes tomaron nota de los hechos observados. Al día siguiente, en el marco de una prueba de autoevaluación, se introdujeron, para los grupos A y B, respectivamente, las siguientes actividades.

\begin{abstract}
Alternativa 1 (grupo A)
En una de las últimas clases visteis un experimento en el que tres tubos conteniendo, respectivamente, (1)1-propanol, (2) propilenglicol (1,2-propanodiol) y (3) glicerina (1,2,3-propanotriol) y una burbuja de aire, ésta ascendía con mayor rapidez en el caso (1), después en el (2) y por último, mucho más lentamente, en (3). Propón una explicación razonada a esta diferencia.
\end{abstract}

(cc) EY-NC-ND 2016, Universitat Politècnica de València 


\section{Alternativa 2 (grupo B)}

En una de las últimas clases visteis un experimento en el que tres tubos conteniendo, respectivamente, (1)1-propanol, (2) propilenglicol (1,2-propanodiol) y (3) glicerina (1,2,3-propanotriol) y una burbuja de aire, ésta ascendía con mayor rapidez en el caso (1), después en el (2) y por último, mucho más lentamente, en (3). A partir de los datos de la tabla siguiente, propón una explicación razonada a esta diferencia.

Tabla 1. Información proporcionada en el modelo de actividad aplicado en el grupo B

\begin{tabular}{|c|c|c|c|}
\hline \begin{tabular}{|l} 
Sustancia \\
\end{tabular} & Estructura molecular & Ta ebullición & \begin{tabular}{|l} 
Viscosidad \\
cinemática(Pa.s)
\end{tabular} \\
\hline 1-propanol & $\mathrm{H}_{3} \mathrm{C}$ & $97^{\circ} \mathrm{C}$ & $2.26 \times 10^{-3}$ \\
\hline \begin{tabular}{|l} 
Propilenglicol \\
(1,2- \\
propanodiol)
\end{tabular} & & $188^{\circ} \mathrm{C}$ & $5.81 \times 10^{-2}$ \\
\hline \begin{tabular}{|l|} 
Glicerina \\
$(1,2,3-$ \\
propanotriol)
\end{tabular} & 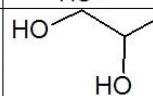 & $290^{\circ} \mathrm{C}$ & 1.5 \\
\hline
\end{tabular}

En el primer caso, los alumnos explicitan y desarrollan sus concepciones sin acceder a más información que la proporcionada durante la realización del experimento en clase. En el segundo caso, pueden contrastar sus puntos de vista iniciales con información explícita acerca de la estructura molecular, así como a los valores de dos propiedades físicas relacionadas con las interacciones moleculares (enlace de hidrógeno, en este caso). La retroalimentación es inmediata y se proporciona a través de un objeto de aprendizaje (polimedia) integrado en la secuencia didáctica (Muñoz, 2006) (https://www.youtube.com/watch?v=2EiqBKkJLX0). Además de proporcionar la solución correcta, explica detalladamente la formación e influencia del enlace de hidrógeno, así como del significado físico de la viscosidad y su relación con hechos de la vida cotidiana. También aclara la confusión lingüística derivado del uso cotidiano del término "densidad" referido a la propiedad física viscosidad. Dos meses más tarde se llevó a cabo el examen de la unidad 1 . Un $15 \%$ de la calificación de este examen estuvo dedicada a la interpretación de las propiedades físicas a partir de la interacciones moleculares. Concretamente, se preguntó acerca de:

a) Diferencias entre las temperaturas de ebullición

b) Diferencias de solubilidad en agua y disolventes orgánicos

c) El mismo experimento estudiado en la actividad, con diferente formato

d) La justificación de las propiedades tensoactivas de un detergente a partir de su estructura molecular.

Se evaluó comparativamente la puntuación conseguida en este bloque del examen, tanto entre los estudiantes que realizaron los dos modelos de actividad y entre estos y los que no la realizaron. La significación de las diferencias entre los valores medios de cada colectivo se evaluó con el estadístico "t" de Student (bloques sombreados en el diagrama de la figura 4), mediante la aplicación Statgraphics Centurion XVI.

(cc) EY-NC-ND 2016, Universitat Politècnica de València

Congreso In-Red (2016) 
Es importante precisar que el diseño de la experimentación vino condicionado por la organización académica del curso, dividido en dos grupos, asignando a cada uno un modelo de actividad, excluyendo por tanto la constitución formal de un tercer grupo control en el que no se realizara. Por este motivo, el grupo control quedó definido por el alumnado que no realizó la actividad, dado su carácter voluntario. Es evidente que este hecho podría haber introducido un sesgo en cuanto a las capacidades de cada colectivo de estudiantes. Por este motivo, se compararon sus puntuaciones en la prueba inicial.

\section{Elaboración de una rúbrica para la evaluación de la actividad}

A partir del análisis cualitativo de las respuestas a la actividad se diseñó una rúbrica que fue aplicada tanto a dichas respuestas, como a la pregunta correspondiente del examen final. Dicho análisis permitió establecer un conjunto de 7 categorías que sirvieron como base para definir los descriptores de la rúbrica utilizada para evaluar la actividad. Estas categorías, con algún ejemplo que clarifica su significado, son enumeradas a continuación:

1. Respuestas correctas basadas en la alusión explícita al enlace de hidrógeno "La diferencia en la velocidades de los tubos es debida a la viscosidad de las sustancias; la viscosidad es la resistencia a fluir que es debido a la fricción interna. En este experimento teníamos tres alcoholes de los cuales se sabe que gracias a los enlaces $\mathrm{O}-\mathrm{H}$ se forman unas fuerzas intermoleculares altas. El 1 propanol al poseer un solo enlace $\mathrm{O}-\mathrm{H}$ tiene menos posibilidades de formar estos puentes de hidrógeno, mientras que por el contrario en este experimento la glicerina al poseer tres grupos hidroxilos tiene mayor posibilidad de formar puentes de hidrógeno".

2. Se refiere exclusivamente a las interacciones moleculares en general, sin citar el enlace de hidrógeno.

"Por la diferencia de fricción interna en las sustancias debido a las fuerzas intermoleculares que en el caso de la glicerina son más fuertes".

3. Cita los grupos hidroxilo como origen del enlace de hidrógeno (explícita o implícitamente, cuando indica su número).

“...la presencia de más $\mathrm{OH}$ hará que se formen más puentes de hidrógeno, y por tanto que las fuerzas intermoleculares sean más fuertes (podemos comprobarlo con la temperatura de ebullición, $1<2<3$ )".

4. Cita exclusivamente el término "alcohol" o "alcoholes" en referencia a los grupos hidroxilo.

“...la cantidad de grupos alcoholes transfiere una viscosidad y un aumento en la temperatura. de ebullición, debido a la posibilidad de formar puentes de hidrógeno, ..."

5. Cita el término "viscosidad" o se refiere a su significado físico como resistencia a fluir 
"Este hecho es debido a la viscosidad, es decir, la medida de la dificultad que tienen los líquidos para fluir como consecuencia de la fricción interna. Esta viscosidad viene determinada por las fuerzas intermoleculares de las sustancias".

6. Cita el término "densidad" como sinónimo erróneo de "viscosidad"

"Cuantos más grupos hidroxilo contenga una sustancia, más puentes de hidrógeno formará dentro de ella. Esto causará que la sustancia sea más densa y la burbuja tarde más en ascender, lo que explica la diferencia de velocidad entre 3,2 y 1 ",

7. Incorpora errores conceptuales relacionados con la naturaleza del hecho experimental observado: diferencia de masa molar como causa, mayor o menor tendencia a la flotación según la densidad relativa de los tres líquidos, unión o atracción con el aire o el oxígeno, etc.

“El aire subia por diferencia de densidades. Cada tubo contenía líquido con una cantidad de grupos alcohol diferente. Como los grupos alcohol interaccionan mucho con el aire apareciendo muchas fuerzas intermoleculares que atraen ciertos componentes del aire como el oxigeno o el nitrógeno".

"La presencia de $\mathrm{OH}$ en la molécula hace que su viscosidad aumente, al igual que lo hace su temperatura de ebullición y fusión y por tanto le cuesta más desplazarse por el tubo, es decir, cuantos más $\mathrm{OH}$ mas interacción con el $\mathrm{O}_{2}$ y más lento es el avance de la molécula".

"Como se observa a medida que se tienen más grupos hidroxilo mayor es la lentitud, ya que el aumento de masa molar que proporciona, hace que sean más pesados cada vez y por lo tanto más lentos".

"Porque $1<2<3$ en cuanto a número de grupos alcoholes. La densidad de los alcoholes aumenta con el número de carbonos y sus ramificaciones. Los alcoholes alifáticos son menos densos que el agua. Es por ello que la burbuja del tubo 1 asciende más rápida que la del 2 y el 3 ”.

Los descriptores de la rúbrica se establecen entonces mediante distintas combinaciones de las anteriores categorías. Es evidente la imposibilidad de un ordenamiento exhaustivo de la calidad de estas combinaciones, lo que daría lugar a una escala de valoración mucho más amplia. En este caso se han configurado según una escala de 0 a 5, tal como se muestra en la tabla 2 .

La innovación aportada en el diseño de la rúbrica utilizada en este trabajo pretende dotar de mayor rigor a la definición de los descriptores. Es importante puntualizar que en este tipo de tareas, en las que las concepciones alternativas de los estudiantes constituyen un elemento decisivo, su definición apriorística a partir de opiniones o categorías generales no vinculadas al contenido específico de la actividad, puede llevar enunciados poco significativos y útiles. Obviamente, esta consideración se refiere exclusivamente al diseño de la rúbrica aplicada en la experimentación, ya que al no ser conocida previamente por el estudiante para saber cómo va a ser evaluado, no es aplicable directamente a la docencia ordinaria. Sin embargo, la definición de sus descriptores puede ser muy útil a la hora de

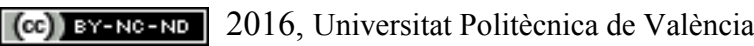

Congreso In-Red (2016) 
orientar el diseño de rúbricas sobre contenidos similares, de modo que el profesorado que aplica las aplica disponga de criterios claros y bien fundamentados para la inclusión de las respuestas de los estudiantes en los descriptores definidos.

Tabla 2. Definición de los descriptores de la rúbrica según las diferentes combinaciones de categorías establecidas a partir del análisis cualitativo de las respuestas

\begin{tabular}{ll}
\hline Puntuación & Definición de los descriptores a partir de las categorías establecidas \\
\hline 5 & $(1+3),(1+3+5)^{*}$ \\
4 & $(1+4),(2+3),(3+5),(1+4+5),(2+3+5),(1+3+6),(2+3+6)$ \\
3 & $1,3,(2+4),(1+6),(3+6),(2+4+6),(1+5),(3+5)$ \\
2 & $2,4,5,(2+6),(4+6),(2+5),(4+5),(2+4+5)$ \\
1 & $6,(1$ a $5+7)$ \\
0 & $7,(6+7)$
\end{tabular}

*La inclusión del término "viscosidad" no ha sido considerada a efectos de calificación por formar parte de la redacción de la actividad en uno de los grupos.

Mediante esta rúbrica se determinaron las diferencias, tanto entre los estudiantes que habían realizado la actividad y los que no lo hicieron, como entre los que la realizaron a través de cada uno de los modelos. A partir de estos datos también se calculó para cada estudiante la diferencia de rendimiento entre ambas pruebas.

\section{Resultados}

Los resultados obtenidos se muestran en la tabla 3, donde las celdas sombreadas muestran los datos cuyas diferencias entre las medias son estadísticamente significativas $(\mathrm{P}<0.05)$

Tabla 3. Valores promedio y significación de las diferencias en las distintas pruebas evaluadas.

\begin{tabular}{|c|c|c|c|c|c|c|c|c|c|c|c|c|c|}
\hline & & \multicolumn{12}{|c|}{ PRUEBA EVALUADA Y PUNTUACIÓN MÁXIMA } \\
\hline & \multirow{2}{*}{ 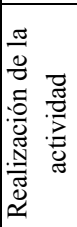 } & \multicolumn{2}{|c|}{$\begin{array}{c}\text { Prueba inicial } \\
\text { (4) }\end{array}$} & \multicolumn{2}{|c|}{ Actividad (5) } & \multicolumn{2}{|c|}{$\begin{array}{l}\text { Pregunta } \\
\text { sobre la } \\
\text { actividad } \\
\text { (exa.) (5) }\end{array}$} & \multicolumn{2}{|c|}{$\begin{array}{c}\text { Diferencia } \\
\text { entre las dos } \\
\text { anteriores }\end{array}$} & \multicolumn{2}{|c|}{$\begin{array}{c}\text { Interpretación } \\
\text { prop. físicas } \\
\text { (exa.) }(15)\end{array}$} & \multicolumn{2}{|c|}{$\begin{array}{c}\text { Total examen } \\
\text { (10) }\end{array}$} \\
\hline & & $\bar{x}$ & $\mathrm{P}$ & $\overline{\mathrm{x}}$ & $\mathrm{P}$ & $\bar{x}$ & $\mathrm{P}$ & $\bar{x}$ & $\mathrm{P}$ & $\bar{x}$ & $\mathrm{P}$ & $\bar{x}$ & $\mathrm{P}$ \\
\hline \multirow{2}{*}{ H } & Sí & 2.30 & \multirow{2}{*}{0.96} & 2.70 & & 3.08 & \multirow{2}{*}{0.47} & 0.39 & & 9.95 & \multirow{2}{*}{0.002} & 6.04 & \multirow{2}{*}{0.17} \\
\hline & No & 2.32 & & & 0.88 & 2.72 & & & 0.81 & 6.28 & & 5.32 & \\
\hline \multirow{2}{*}{$\mathrm{H}$} & Sí & 1.94 & \multirow{2}{*}{0.76} & 2.78 & & 3.41 & \multirow{2}{*}{0.03} & 0.53 & & 8.87 & \multirow{2}{*}{0.11} & 6.01 & \multirow{2}{*}{0.04} \\
\hline & No & 2.06 & & & & 2.06 & & & & 6.51 & & 4.91 & \\
\hline \multirow{2}{*}{$\frac{n}{+}$} & Sí & & & & & 3.23 & \multirow{2}{*}{0.04} & & & 9.49 & \multirow{2}{*}{0.001} & 6.03 & \multirow[b]{2}{*}{0.02} \\
\hline & $\mathrm{No}$ & & & & & 2.43 & & & & 6.38 & & 5.14 & \\
\hline
\end{tabular}


Tal como ya se indicó anteriormente, las puntuaciones de la prueba inicial no muestran diferencias significativas entre los colectivos que realizaron o no la actividad, en cada uno de los grupos. Este resultado garantiza de algún modo la homogeneidad de los grupos establecidos al interpretar el resto de resultados obtenidos.

Cabe destacar la presencia de diferencias estadísticamente significativas al considerar cómo la realización de la actividad (independientemente del modelo utilizado) influye tanto en la tarea del examen relacionada con la misma, como, sobre todo, en el bloque del examen dedicado a la interpretación de las propiedades físicas a partir de las interacciones moleculares. Si distinguimos los modelos de actividad, el grupo A (planteamiento abierto) muestra un claro y significativo incremento en dicho bloque del examen, mientras que es precisamente en este grupo donde no hay diferencias significativas entre quienes realizaron o no la actividad, con respecto a la puntuación total del examen. Este resultado podría considerarse una obviedad si el contenido evaluado en el examen hubiera estado referido exclusivamente a la actividad experimentada. El alcance de este resultado reside precisamente en el grado en que el aprendizaje adquirido a través de dicha actividad ha sido transferido a otros contextos relacionados con la interpretación de las propiedades físicas a partir de las interacciones moleculares.

Dentro del colectivo que realizó la actividad, al comparar para cada individuo la puntuación obtenida con la de la tarea del examen relacionada con ella, en ambos modelos se observa un incremento que puede interpretarse como un efecto positivo de la retroalimentación, aunque bastante moderado. No obstante, también cabe valorar el hecho de la persistencia de dicho efecto, ya el examen tuvo lugar dos meses después de la actividad. En este caso, aunque sin tratarse de una diferencia estadísticamente significativa, el incremento individual de la puntuación es mayor en el modelo B.

\section{Conclusiones}

Valorando globalmente los resultados obtenidos, y asumiendo las limitaciones propias de una innovación aplicada en el contexto de la docencia habitual del curso, puede admitirse que la realización de la actividad experimentada mejoró significativamente la capacidad de los estudiantes para interpretar correctamente los cambios físicos a partir de las interacciones moleculares. Sin embargo, no parece existir una influencia clara del modo de plantear la actividad, por lo que el efecto positivo observado cabría atribuirlo principalmente a la retroalimentación aplicada, que sí fue común a ambos grupos.

En cuanto a los objetivos de tipo metodológico, la definición de los descriptores de una rúbrica puede ser mucho más rigurosa y sobre todo más fácil de aplicar cuando se fundamenta en un análisis cualitativo previo de la actividad. Este puede considerarse particularmente útil en aquellas tareas, tan típicas del aprendizaje científico, donde es frecuente en los estudiantes la presencia de esquemas conceptuales alternativos. Por otra parte, cabe destacar la potencialidad de objetos de aprendizaje como los "polimedias" a la hora de proporcionar retroalimentación. En este sentido, sería importante profundizar en el conocimiento de cómo los estudiantes utilizan realmente estos recursos al mismo tiempo que lograr una mayor interactividad en su aplicación.

(cc) EY-NC-ND 2016, Universitat Politècnica de València

Congreso In-Red (2016) 
Por último, cabe aludir también a las dificultades inherentes a la evaluación experimental de innovaciones didácticas sin distorsionar el trabajo habitual del aula. En este sentido, cabe reivindicar una vez más la necesidad de convertir la docencia ordinaria en una investigación sobre la práctica, debidamente organizada y valorada.

\section{Referencias}

BURTON, G., HOLMAN, J., PILling, G., WADDINGTON, D. (1994). Salters Advanced Chemistry. Chemical Ideas. Heinemann, Oxford.

COPPOLA, B. P. (1995) "Progress in Practice: Using Concepts from Motivational and SelfRegulated Learning Research to Improve Chemistry Instruction". New directions for teaching and learning, 63. (Fall 1995), p. 87-96.

DRIVER, R. (1986). Psicología cognoscitiva y esquemas conceptuales de los alumnos. Enseñanza de las Ciencias, 4(1), p. 3-15.

GALAGOVSKY, L. R., GIACOMO, M. A. D., Y CASTELO, V. (2009). "Modelos vs. dibujos: el caso de la enseñanza de las fuerzas intermoleculares". Revista electrónica de enseñanza de las ciencias, 8(1), 1 .

GARCÍA-JIMÉNEZ, E. (2015). "La evaluación del aprendizaje: de la retroalimentación a la autorregulación. El papel de las tecnologías". RELIEVE-Revista Electrónica de Investigación y Evaluación Educativa, 21(2), p. 1-24.

GILBERT, J. K. Y TREAGUST, D. F. (2009). "Introduction: Macro, submicro and symbolic representations and the relationship between them: Key models in chemical education", en Multiple representations in chemical education (p. 1-8). Springer Netherlands.

GONZÁLEZ, R. R. (2006). "Diseño de entornos para el desarrollo de la autonomía en el aprendizaje”. Aula abierta, 87, p. 89-103.

HENDERLEITER, J., SMART, R. ANDERSON, J. Y ELIAN, O. (2001). "How do organic chemistry students understand and apply hydrogen bonding?" Journal of Chemical Education, 78(8), p. 1126.

KIND, V. (2004). "Beyond Appearances: Students' misconceptions about basic chemical ideas". School of Education, Durham University.

$<\underline{\text { http://citeseerx.ist.psu.edu/viewdoc/download?doi=10.1.1.649.3454\&rep=rep1\&type }=\text { pdf }>}$ [consulta: 2 de abril de 2016]

KRONIK, L., NAHUM, T.L., MAMLOK-NAAMAN, R. Y HOFSTEIN, A. (2008). "A new" bottomup" framework for teaching chemical bonding”. Journal of Chemical Education, 85 (12), p. 1680.

NAHUM, T. L., MAMLOK-NAAMAN, R., HOFSTEIN, A., Y TABER, K. S. (2010). "Teaching and learning the concept of chemical bonding”. Studies in Science Education, 46(2), p. 179-207.

LLORENS, J. A. (1991). Comenzando a aprender química. Ideas para el diseño curricular. Visor. Madrid.

MALDONADO, R. (2009). "Sobre la retroalimentación o el feedback en la educación superior on line”. Revista Virtual Universidad Católica del Norte, 26, p. 1-18. 
$<\underline{\text { http://revistavirtual.ucn.edu.co/index.php/RevistaUCN/article/viewFile/117/230 }}>$ [Consulta: 2 de abril de 2016]

MARTÍN, M. G. (2012). “Academic self-regulation as an explanatory variable of university learning processes”. Profesorado. Revista de Currículum y Formación del Profesorado, 16(1), p. 203-221.

$<$ http://recyt.fecyt.es/index.php/profesorado/article/viewFile/48970/30170> [consulta: 2 de abril de 2016]

MUÑOZ ARTEAGA, J., ÁlVAREZ RODRÍGUEZ, F. J., OSORIO URRUTIA, B., Y CARDONA SALAS, J. P. (2006). "Objetos de aprendizaje integrados a un sistema de gestión de aprendizaje”. Apertura, 6(3), p.109-117.

NAHUM, T. L., MAMLOK-NAAMAN, R., HOFSTEIN, A., Y KRAJCIK, J. (2007). "Developing a new teaching approach for the chemical bonding concept aligned with current scientific and pedagogical knowledge". Science Education, 91(4), p. 579-603.

ÖZMEN, H. (2004). "Some student misconceptions in chemistry: A literature review of chemical bonding". Journal of Science Education and Technology, 13(2), p. 147-159.

PETRUCCI, R.H., HARWOOD, W.S. Y HERRING, F.G. (2010). Química General. Pearson Educación, Madrid (España)

POZO, J. I. (1991). Procesos cognitivos en la comprensión de la ciencia: las ideas de los adolescentes sobre la química (Vol. 65). Ministerio de Educación. Madrid (España).

SCHMIDT, H. J., KAUFMANN, B., Y TREAGUST, D. F. (2009). "Students' understanding of boiling points and intermolecular forces". Chemical Education Research and Practice, 10(4), p. 265272.

TABER, K. S. (2001). "Building the structural concepts of chemistry: Some considerations from educational research". Chemical Education Research and Practice, 2(2), p. 123-158.

TALANQUER, V. (2009). "On cognitive constraints and learning progressions: The case of "structure of matter". International Journal of Science Education, 31(15), p. 2123-2136.

TARHAN, L., AYAR-KAYALI, H., UREK, R. O., Y ACAR, B. (2008). "Problem-based learning in 9th grade chemistry class: 'Intermolecular forces'”. Research in Science Education, 38(3), p. 285-300.

WEINHOLD, F. Y KLEIN, R. A. (2014). "What is a hydrogen bond? Resonance covalency in the supramolecular domain”. Chemistry Education Research and Practice, 15(3), 276-285.

VYGOTSKY, L. S. (1993). Obras escogidas (II): Pensamiento y Lenguaje, Conferencias sobre Psicología. Visor Aprendizaje, Madrid.

(cc) EY-NC-ND 2016, Universitat Politècnica de València

Congreso In-Red (2016) 